\title{
Relativistic Bi-Stability in a Plasma Beatwave Accelerator
}

\author{
Gennady Shvets
}

\begin{abstract}
Department of Physics and Institute for Fusion Studies, The University of Texas at Austin, Austin,
\end{abstract} TX 78712

\begin{abstract}
Beatwave excitation of plasma waves is analyzed in the regime where relativistic corrections to the plasma frequency are important. It is shown that a long beatwave pulse can excite strong plasma waves in its wake even when the beatwave frequency is detuned from the electron plasma frequency. The wake is caused by the dynamic bi-stability of the nonlinear plasma wave if the beatwave amplitude exceeds the analytically calculated threshold. Two possible beatwave drivers are considered: intensity-modulated laser pulse and density-modulated electron beam. It is found that, due to the relativistic bi-stability, so portions of the driver may experience photon blue-shifting (for the laser driver) or electron acceleration (for the beam driver). In the latter case a combined accelerator/injector is envisioned.
\end{abstract}

\section{INTRODUCTION}

Although plasma beatwave accelerator (PBWA) is one of the original advanced acceleration concepts utilizing plasma [1], it continues attracting significant experimental and theoretical attention [2,3][4] as a basic nonlinear plasma phenomenon, and as a viable approach to plasma-based particle acceleration. Beatwave excitation of the electron plasma waves is realized when the driver intensity (laser or particle beam) is modulated at the frequency $\omega_{B}$ close to that of the plasma wave. In the linear regime electron plasma wave behaves as a linear oscillator which is most effectively excited when $\omega_{B}=\omega_{p}$ where, $\omega_{p}=\sqrt{4 \pi e^{2} n_{0} / m}$ is the electron plasma frequency, $-e$ and $m$ are the electron charge and mass, and $n_{0}$ is the plasma density. Plasma wave can be driven offresonance with $\omega_{B} \neq \omega_{p}$, but higher beatwave intensity is required [3]. A finite-duration beatwave can excite a plasma wave in its wake only if its duration $\tau_{L}<\pi /|\Delta \omega|$, where $\Delta \omega \equiv \omega_{B}-\omega_{p}$ is the frequency detuning.

Plasma wave becomes nonlinear when the plasma velocity becomes relativistic. The plasma wave frequency becomes dependent on its amplitude through the relativistic mass increased: $\omega_{p} \rightarrow \omega_{p} / \gamma$, where $\gamma$ is the relativistic factor. Rosenbluth and Liu [5] demonstrated that this dependency results in the relativistic saturation of the plasma wave amplitude. A number of authors have proposed ways of circumventing the Rosenbluth-Liu limit by detuning $[6,7]$ or chirping [8] the beatwave frequency of long drivers.

The focus of this paper is the plasma response to a finite-duration detuned beatwave pulse in the regime where relativistic corrections are important. Specifically, it is shown that a strong plasma wave can be excited in the wake of a relatively long beatwave pulse 
of duration $t_{L} \gg 1 / \Delta \omega$ due to the nonlinear phenomenon of dynamic relativistic bistability (RB) [9]. Another manifestation of the RB is that, at a certain critical strength of the beatwave driver, a weak driven plasma wave becomes unstable, and a much higher amplitude wave is excited. Linear estimates of the plasma wave amplitude fail when the beatwave amplitude exceeds this detuning-dependent critical strength. As the time-dependent beatwave strength increases and exceeds the critical value, significant pulsations of the plasma wave amplitude occur. These pulsations indicate that significant energy exchange takes place between the plasma wave and the driver. This effect can be exploited when the beatwave is a microbunched electron beam [10] which can be produced, for example, by an inverse free-electron laser. Plasma wave is then driven by the bunches in the head of the beam, while those in the back deplete plasma the plasma wave, thereby gaining energy.

Relativistic bi-stability was originally described [9] for a magnetized electron subjected to cyclotron heating. Applications of RB to electron cyclotron heating of fusion plasmas $[11,12]$ have been later suggested. But the RB of the beatwave-driven plasma wave has never been explored, either as a basic phenomenon or in the context of plasmabased accelerators.

\section{DERIVATION OF THE BASIC EQUATIONS AND STEADY STATE ANALYSIS}

The one-dimensional relativistic dynamics of the cold plasma driven by a beatwave can be described using the Lagrangian displacement of the plasma element originally located at $z_{0}: z(t)=z_{0}+\zeta\left(t, z_{0}\right)$. It is assumed that the beatwave generated by either a pair of frequency-detuned laser beams, or a modulated electron beam, is moving with the speed close to the speed of light $c$, and, therefore, all beatwave quantities are functions of the co-moving coordinate $\tau^{\prime}=\omega_{p}(t-z / c) \equiv \tau-\omega_{p} \zeta / c$. Introducing the normalized displacement $\tilde{\zeta}=\omega_{p} \zeta / c$ and longitudinal relativistic momentum $\tilde{p}=\gamma d \tilde{\zeta} / d \tau$, where $\gamma=\sqrt{1-\vec{v}^{2} / c^{2}}$, equations of motion take on the form

$$
\frac{d \tilde{\zeta}}{d \tau}=\frac{\tilde{p}}{\sqrt{1+\tilde{p}^{2}}}, \quad \frac{d \tilde{p}}{d \tau}=-\tilde{\zeta}+a\left(\tau^{\prime}\right) \cos \omega \tau^{\prime} .
$$

Assuming that $|\Delta \omega| \ll \omega_{p}$ (near-resonance excitation), transverse momentum of the plasma has been neglected and the relativistic $\gamma$-factor simplified to $\gamma=\sqrt{1+\tilde{p}^{2}}$. The first term in the force equation is the restoring force of the ions, and the second term signifies the beatwave with the frequency $\omega_{B} \equiv \omega \omega_{p}$. The nonlinear in $\zeta$ modification of the beatwave in the rhs of Eqs. (1) is neglected in what follows. For a pair of linearly polarized laser pulses with electric field amplitudes $E_{1}$ and $E_{2}$ and the corresponding frequencies $\omega_{1}$ and $\omega_{2}=\omega_{1}-\omega_{B}$ the normalized beatwave amplitude $a=(e / m c)^{2} E_{1} E_{2} / 2 \omega_{1} \omega_{2}$ [5]. For a driving electron bunch with the density profile $n_{b}=n_{b 0}+\delta n_{b} \sin \omega \tau$ it can be shown that $a=\delta n_{b} / n_{0}$. Although arbitrary profiles of $a(\tau)$ are allowed, it is assumed that $|d a / d \tau| \ll|a|$. The total energy density of the plasma wave $U_{p} / n_{0} m c^{2}=\sqrt{1+\tilde{p}^{2}}+\tilde{\zeta}^{2} / 2$ is changed via the interaction with the beatwave. The 
effect of the plasma wave on the beatwave is neglected for the moment and addressed in Section .

Although Eqs. (1) can be solved numerically at this point, further simplification is made by assuming $\tilde{p}=u \cos (\omega \tau+\phi)$, where $u$ and $\phi$ are slowly varying functions of $\tau$. In the weakly relativistic approximation $\tilde{p}^{2} \ll 1$ obtain:

$$
\begin{aligned}
& \frac{d u}{d \tau}=\frac{a}{2} \cos \phi \\
& u \frac{d \phi}{d \tau}=-\frac{a}{2} \sin \phi-\frac{u}{2 \omega}\left(\omega^{2}-1+3 u^{2} / 8\right) .
\end{aligned}
$$

We can now find the equilibrium points of Eq. (2), $d u / d \tau=0$ (steady amplitude), and Eq. (3), $d \phi / d \tau=0$ (phase-locking to the beatwave). Their number (one or three) depends on the beatwave frequency $\omega$ and the amplitude $a$. For any $\omega$ there is a stable equilibrium point: $\phi_{0}=-\pi / 2$ and $u_{0}>0$ found as the root of the third-order polynomial equation $\mathscr{P}\left(u_{0}\right)=u_{0}\left(\omega^{2}-1+3 / 8 u_{0}^{2}\right)=\omega a$. For the most interesting $\omega<1$ regime additional solutions $\phi_{0}=\pi / 2$ and $u_{0}>0$, where $u_{0}$ is the positive root of $\mathscr{P}$ $\left(u_{0}\right)=-\omega a$, may be found, depending on the beatwave amplitude. Specifically, there are no additional positive roots for $a>a_{\text {crit }}$, where $a_{\text {crit }}=4 \sqrt{2}\left(1-\omega^{2}\right)^{3 / 2} / 9 \omega$, and two positive roots $u_{1,2}$ for $a<a_{\text {crit }}$ (one of them unstable). Stable equilibrium amplitudes $u_{0}$ with $\phi_{0}=\pi / 2$ (Branch 1) and $\phi_{0}=-\pi / 2$ (Branch 3), as well as the unstable one (Branch 2) are plotted in Fig. 1 as a function of the beatwave strength $a$ for $\omega=0.95$ $\left(a_{\text {crit }}=0.02\right)$.

Equilibrium bi-stability corresponding to Branches 1 and 3 is universal for any nonlinear pendulum $[13,9]$, including a weakly damped one. Equilibrium solutions are meaningful only if the plasma wave is phase-locked to the beatwave: $d \phi / d \tau \approx 0$. As shown below, this is not the case when the peak beatwave amplitude exceeds $a_{\text {crit }}$. Nonetheless, a dynamic RB described below occurs even in the absence of phase-locking.

Remarkably, there are two solutions for $a=0-$ the trivial one $u_{1}=0$, and the nontrivial $u_{2}=2 \sqrt{2\left(1-\omega^{2}\right) / 3}$ corresponding to the plasma wave left in the wake of a detuned beatwave pulse. In the non-impulsive regime of long pulses with $\tau>1 /\left|1-\omega^{2}\right|$ the only way of exciting a non-vanishing wake is through the relativistic bi-stability effect described in this Letter.

Equilibrium solutions described above and plotted in Fig. 1 are useful guidelines in determining the evolution of $(u, \phi)$ in response to a slowly-changing beatwave amplitude $a(\tau)$. For example, the dot-dashed line in Fig. 1 shows steady-state plasma response for zero detuning $(\omega=1)$. In that case plasma wave amplitude responds to a smooth Gaussian beatwave pulse by increasing first, reaching the maximum, and then decreasing to zero after the pulse. Plasma wave remains phase-locked to the beatwave, and therefore the equilibrium solution is meaningful. Because there is only one equilibrium solution for $\omega \geq 1$, no relativistic bi-stability is expected. The situation is very different for $\omega<1$. The solid-line branches 1 and 2 correspond to the stable steady-state response for $\omega=0.95$, the dashed-line branch 2 is unstable. Nonlinear bi-stability for $a<a_{\text {crit }}=0.02$ is apparent: there are two stable equilibria available to the system. Which one of the two is physically accessible depends on the total time history of the system. 


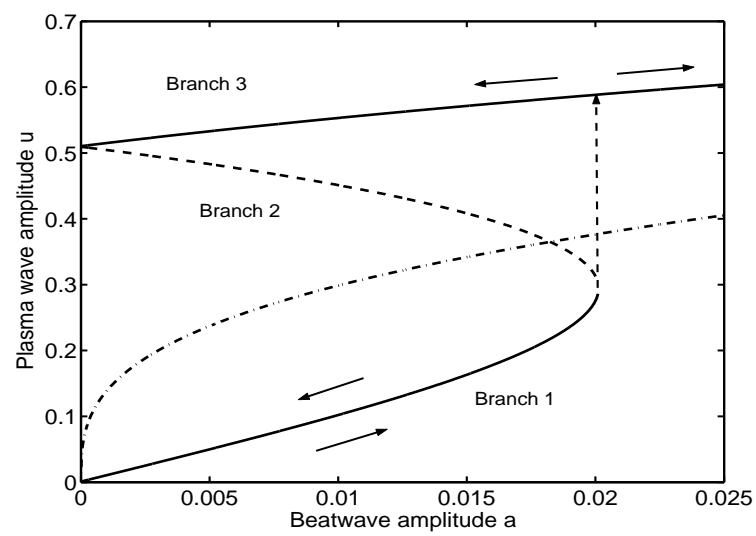

FIGURE 1. Steady-state solutions of a driven plasma wave as a function of the beatwave amplitude $a$. Solid lines 1, 2: stable equilibria for $\omega=0.95$; dashed line: unstable equilibrium for $\omega=0.95$; dot-dashed line: resonant excitation with $\omega=1$.

\section{Wake excitation by a Gaussian beatwave pulse}

Consider plasma response to a Gaussian beatwave pulse $a(\tau)=a_{0} \exp \left(-\tau^{2} / \tau_{L}^{2}\right)$, where $\tau_{L} \gg 1 /|1-\omega|$ is the normalized pulse duration. Although the presented above steady-state analysis is strictly applicable only for $a(\tau)=$ const, we'll find that the equilibrium solutions are followed when the beatwave amplitude is small, and its rate of change slow. For $a_{0}<a_{\text {crit }}$ the plasma response is as follows: amplitude $u$ adiabatically follows $a(\tau)$ by staying on the Branch 1 and following the equilibrium trajectory schematically shown by arrows in Fig. 1 . The adiabaticity condition is $\Omega_{B} \tau_{L} \gg 1$, where $\Omega_{B}$ is the bounce frequency around the equilibrium point $u_{0}$ such that $\mathscr{P}\left(u_{0}\right)=-\omega a(\tau)$. Linearizing Eqs. $(2,3)$ around $\phi=\pi / 2$ and $u=u_{0}$ yields $\Omega_{B}^{2}=a\left(u_{c r i t}^{2}-u_{0}^{2}\right) / 4 \omega u_{0}$, where $u_{\text {crit }}=2 \sqrt{2\left(1-\omega^{2}\right)} / 3$ is the critical plasma wave amplitude corresponding to the merging point between Branches 1 and 2 in Fig. 1. For $a_{0}<a_{\text {crit }}$ plasma oscillation is indeed phase-locked to the beatwave at $\phi_{0} \approx \pi / 2$ during the ramp-up and most of the ramp-down of the laser pulse (although phase-locking is lost when the pulse amplitude becomes very small on the down-ramp). As the result, plasma wave amplitude returns to a very small value in the wake of the beatwave, as shown by a dot-dashed line in Fig. 2. The longer is the beatwave pulse duration $\tau_{L}$, the smaller is the wake because its non-vanishing amplitude is due to the adiabaticity violation for finite $\tau_{L}$.

For example, for example, for $a=0.01, u_{0}=0.1$, and $\omega=0.95$ obtain $\Omega_{B} \approx 0.04$, and the adiabaticity requirement is $\tau_{L}>25$.

Situation changes for $a_{0}>a_{\text {crit }}$ : as $a(\tau)$ approaches $a_{\text {crit }}$, the adiabatic condition is violated (noted in the context of electron cyclotron heating [11, 12]), and phase-locking at $\phi_{0}=\pi / 2$ is no longer possible. Thus, the transfer to Branch 3 schematically shown by a vertical arrow in Fig. 1 becomes feasible, and the plasma wave amplitude can dramatically increase. In the presence of a finite plasma wave damping this indeed 


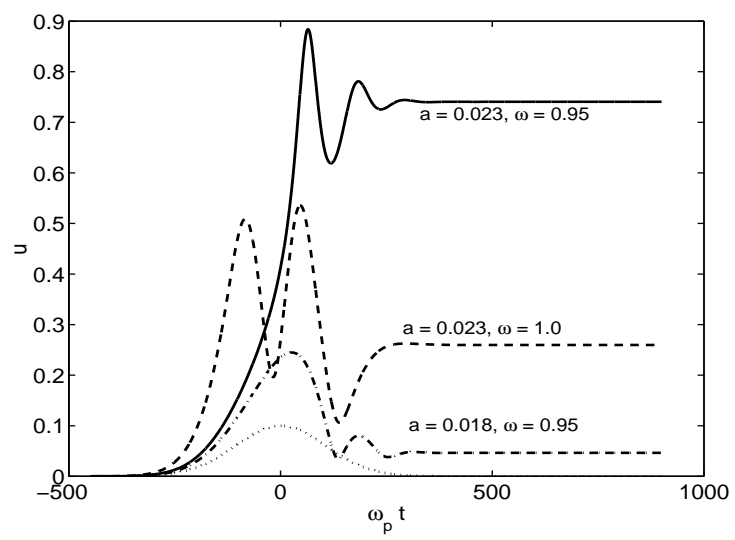

FIGURE 2. Excitation of a plasma wave by a Gaussian beatwave pulse (dotted line), $a(\tau)=$ $a_{0} \exp \left[-\tau^{2} / \tau_{L}^{2}\right], \tau_{L}=150$. Solid line: $\omega=0.95$, above-threshold excitation with $a_{0}=0.023>a_{\text {crit }}=0.02$; dashed line: resonant excitation with $\omega=1$ and $a_{0}=0.023$; dot-dashed line: $\omega=0.95$, below-threshold excitation with $a_{0}=0.018<a_{\text {crit }}$.

happens: the subsequent decrease of the beatwave amplitude results in phase-locking at $\phi_{0}=-\pi / 2$, with $u$ following along the Branch 3 . Without damping, there is no mechanism for the plasma wave to reach the equilibrium amplitude given by the upper Branch 3. As shown below, a conservation law prohibits the jump between Branches 1 and 3.

Nevertheless, even without damping, a significant plasma wave is left behind the finite-duration beatwave pulse (Fig. 2, solid line). The previously unaccessible finiteamplitude solution has been reached due to the effect of the dynamic RB which is best understood through the conservation of the effective Hamiltonian of the driven plasma wave. The effective Hamiltonian

$$
H=\frac{1}{2} a u \sin \phi+\frac{\left(\omega^{2}-1\right) u^{2}}{4 \omega}+\frac{3 u^{4}}{64 \omega}
$$

can be used to express Eqs. $(2,3)$ in the form of $\dot{u}=(1 / u) d H / d \phi, \dot{\phi}=-(1 / u) d H / d u$. For a slowly changing beatwave amplitude $a(\tau)$ the Hamiltonian is almost conserved: $d H / d \tau=0.5 u \sin \phi d a / d \tau \approx 0$. This constitutes the conservation law preventing the jump between Branches 1 and 3. For the initially quiescent plasma $a=0$ and $u=0$ before the arrival of the beatwave. Therefore, $H \approx 0$ after its passage, as confirmed by numerical simulations of various pulse durations and amplitudes. Remarkably, in addition to the trivial quiescent plasma solution $u=0$, there is a second $u_{\infty}=4 \sqrt{\left(1-\omega^{2}\right) / 3}$ solution satisfying $H\left(u_{\infty}\right)=0$. Thus, a plasma wave with $H=0$ is dynamically bi-stable: after the passage of the beatwave it can be either quiescent, or have the finite amplitude $u_{\infty}$. It is conjectured that, by using a beatwave pulse with $a_{0}>a_{c r i t}$, the latter solution can be accessed, thereby leaving a wake of a substantial plasma wave with amplitude $u_{\infty}$. 
This conjecture is verified by numerically integrating Eqs. $(2,3)$ for two different detunings (resonant, with $\omega=1$, and non-resonant, with $\omega=0.95$ ) and two beatwave amplitudes (sub-threshold, with $a_{0}=0.018$, and above-threshold, with $a_{0}=0.023$ ). In all cases the Gaussian pulse duration was chosen $\tau_{L}=150$. In physical units, for the plasma density of $n_{0}=10^{19} \mathrm{~cm}^{-3}$ the corresponding pulse duration is $t_{L} \equiv \tau_{L} / \omega_{p} \approx 750$ fs. Simulation results are shown in Fig. 2, where the solid line corresponds to the most interesting of the three cases: $\omega=0.95$ and $a_{0}=0.023$. The plasma wave amplitude of $u \approx 0.75$ in the wake of the laser pulse is in a good agreement with $u_{\infty}=0.72$. This wake owes its existence to the dynamic RB: upon interacting with the above-threshold laser beatwave, plasma wave is transferred from the quiescent state of $u=0$ to the excited state of $u=u_{\infty}$. The sub-threshold excitation (dot-dashed line) with the same detuning fails to transfer the plasma into the excited state, yielding a negligible wake that is an order of magnitude smaller than in the above-threshold regime.

Linear theory also fails to describe the strong wake in this example because the detuning and the pulse duration are chosen such that the linear prediction $u_{\text {lin }}=a_{0} /(1-$ $\left.\omega^{2}\right) \times \exp \left[-\tau_{L}^{2}(\omega-1)^{2} / 4\right] \approx 0$ is negligibly small. Resonant excitation (dashed line) also yields a much smaller wave. Moreover, the resonantly and the sub-threshold excited plasma waves would have been even smaller had the adiabatic assumption been fully satisfied. Indeed, it is numerically confirmed that the wake amplitudes for the resonant and the sub-threshold excitations rapidly decline for longer pulses, whereas the amplitude of the non-resonant above-threshold excitation is insensitive to the beatwave pulse length $\tau_{L}$.

\section{Driver Deceleration}

So far the effect of the plasma wave on the driver has been neglected. Of course, the energy of the plasma wave is supplied by the beatwave. Since the plasma wave energy changes non-monotonically, different portions of the beatwave either lose or gain energy. In the weakly relativistic case, the plasma energy density $U_{p} \approx n_{0} m c^{2} u^{2} / 2$. For concreteness, I concentrate on the above-threshold case plotted in Fig. 2 (solid line). The leading portion of the beatwave $(-\infty<\tau<64)$ contributes energy to the beatwave and is, therefore, depleted. If the beatwave is produced by a laser pulse, this depletion can be described in the language of photon deceleration, or red-shifting Wilks et al.. In the context of the laser beatwave the red-shifting corresponds to the scattering of the photons from the higher frequency into the Stokes component. Assuming equal amplitude lasers, $E_{1}=E_{2}$, the rate of the frequency shifting (per unit of the propagation length) can be found as $-d \omega / d z \approx\left(\omega_{p}^{3} / 4 c \omega_{1} a\right) \times d\left(u^{2}\right) / d \tau$. Therefore, the laser pulse is red (blue) shifted if $d u / d \tau>0(d u / d \tau<0)$.

If the beatwave is produced by a microbunched electron beam, the sign of $d u / d \tau$ can be related to the acceleration or deceleration gradient of the drive electron bunch $E_{z}$ through

$$
\frac{E_{z}(\tau)}{E_{\mathrm{WB}}}=\frac{\delta n_{b}}{n_{b 0}}\left(\frac{1}{2 a(\tau)} \frac{d u^{2}}{d \tau}\right),
$$


where $E_{\mathrm{WB}}=m c \omega_{p} / e$ is the non-relativistic wavebreaking electric field. Again, the sign of $d u / d \tau$ determines whether the driving bunch is accelerated or decelerated. For a microbunched electron driver consisting of femtosecond bunches with duration $\delta t \ll 1 / \omega_{p}$ [10] produced by an inverse free-electron laser $\delta n_{b} \sim n_{b 0}$. It is estimated that in the plasma wave decay region of the driving bunch $(64<\tau<112)$ the beam is decelerated at a rate of $E_{z} \approx 30 \mathrm{GeV} / \mathrm{m}$ for $n_{0}=10^{19} \mathrm{~cm}^{-3}$. Therefore, the marriage of the microbunched plasma wakefield accelerator and the dynamic relativistic bi-stability concepts yields a new advanced acceleration technique which takes advantage of the temporal drive beam structure to produce high energy femtosecond electron beams.

\section{ACKNOWLEDGMENTS}

Support for this work was provided by the US Department of Energy under Contracts No. DE-FG02-04ER54763 and DE-FG02-04ER41321.

\section{REFERENCES}

1. Tajima, T., and Dawson, J. M., Phys. Rev. Lett., 43, 267 (1979).

2. Joshi, C., and Katsouleas, T., Physics Today, 56, 47 (June 2003).

3. Tochitsky, S. Y., Narang, R., Filip, C. V., Musumeci, P., Clayton, C. E., Yoder, R. B., Marsh, K. A., Rosenzweig, J. B., Pellegrini, C., and Joshi, C., Phys. Rev. Lett., 92, 095004 (2004).

4. Lindberg, R. R., Charman, A. E., Wurtele, J. S., and Friedland, L., Phys. Rev. Lett., 93, 055001 (2004).

5. Rosenbluth, M. N., and Liu, C. S., Phys. Rev. Lett., 29, 701 (1972).

6. Tang, C. M., Sprangle, P., and Sudan, R. N., Phys. Fluids, 28, 1974 (1985).

7. McKinstrie, C. J., and Forslund, D. W., Phys. Fluids, 30, 904 (1987).

8. Deutsch, M., Meerson, B., and Golub, J. E., Phys. Fluids B, 3, 1773 (1991).

9. Kaplan, A. E., Phys. Rev. Lett., 48, 138 (1982).

10. Liu, Y., Wang, X. J., Cline, D. B., Babzien, M., Fang, J. M., Gallardo, J., Kusche, K., Pogorelsky, I., Skaritka, J., and van Steenbergen, A., Phys. Rev. Lett., 80, 4418 (1998).

11. Nevins, W. M., Rognlien, T. D., and Cohen, B. I., Phys. Rev. Lett., 59, 60 (1987).

12. Kotel'nikov, I. A., and Stupakov, G. V., Phys. Fluids B, 2, 881 (1990).

13. Landau, L. D., and Lifshitz, E. M., Mechanics, Pergamon Press, New York, 1976, $3 d$ edn.

14. Wilks, S. C., Dawson, J. M., Mori, W. B., Katsouleas, T., and Jones, M. E., Phys. Rev. Lett., 62, 2600 (1989). 